$\widehat{\underline{\text { IIIIIII }}}$

\title{
GHENT UNIVERSITY
}

biblio.ugent.be

The UGent Institutional Repository is the electronic archiving and dissemination platform for all UGent research publications. Ghent University has implemented a mandate stipulating that all academic publications of UGent researchers should be deposited and archived in this repository. Except for items where current copyright restrictions apply, these papers are available in Open Access.

This item is the archived peer-reviewed author-version of:

Expanded-Beam Backside Coupling Interface for Alignment-Tolerant Packaging of Silicon Photonics

N. Mangal, B. Snyder, J. Van Campenhout, G. Van Steenberge, and J. Missinne

In: IEEE Journal of Selected Topics in Quantum Electronics, 2019.

https://doi.org/10.1109/JSTQE.2019.2934161

To refer to or to cite this work, please use the citation to the published version:

Mangal, N., Snyder, B., Van Campenhout, J., Van Steenberge, G., \& Missinne, J. (2019), Expanded-Beam Backside Coupling Interface for Alignment-Tolerant Packaging of Silicon Photonics. IEEE Journal of Selected Topics in Quantum Electronics, 26(2), 1-7. 


\title{
Expanded-Beam Backside Coupling Interface for Alignment-Tolerant Packaging of Silicon Photonics
}

\author{
Nivesh Mangal, Bradley Snyder, Joris Van Campenhout, Geert Van Steenberge, and Jeroen Missinne
}

\begin{abstract}
We demonstrate an alignment-tolerant backside coupling interface in the $\mathbf{O}$-band for silicon photonics by generating an optimized through-substrate (downward) directionality beam from a TE-mode grating coupler and hybrid integrating the chip with backside silicon microlenses to achieve expanded beam collimation. The key advantage of using such an expanded beam interface is an increased coupling tolerance to lateral and longitudinal misalignment. A $34 \mu \mathrm{m}$ beam diameter was achieved over a combined substrate thickness of $630 \mu \mathrm{m}$ which was then coupled to a thermally expanded core single-mode fiber to investigate the tolerances. A 1-dB fiber-to-microlens lateral alignment tolerance of $14 \mu \mathrm{m}$ and an angular alignment tolerance of $1^{\circ}$ was measured at a wavelength of $1310 \mathrm{~nm}$. In addition, a large $\pm 2.5 \mu \mathrm{m}$ 1-dB backside alignment accuracy was measured for the placement of microlens with respect to the grating. The radius of curvature of $\mathrm{Si}$ microlens to achieve a collimated beam was $480 \mu \mathrm{m}$, and a 1-dB longitudinal alignment tolerance of $700 \mu \mathrm{m}$ was measured for coupling to a single-mode expanded core fiber. The relaxation in alignment tolerances make the demonstrated coupling interface suitable for chip-to-package or chip-to-board coupling.
\end{abstract}

Index Terms-Optical Interposer; Silicon Photonics; Microlenses; Collimator; Expanded Core Fibers; Grating Couplers.

\section{INTRODUCTION}

W ITH a growing need for higher capacity and performance in the datacenters, single-mode optics is projected to surpass the multimode-optics based solutions in the next decade for short-reach distances. Silicon photonics technology will be at the forefront to cater to this demand bringing single-mode transmission, dense integration and wavelength division multiplexing capabilities along with it. There has been a rapid increase in the number of CMOSbased wafer-scale manufacturing fabs providing access to silicon photonics design services [1] and this investment is in sync with the expectation of the volume of silicon photonics based components needed, out of which a significant portion is going to be absorbed in the datacenter market [2]. Packaging is one of the primary contributing factor in increasing the overall cost of a silicon photonics based transceiver [3], [4]. Several processes such as manual assembly, fiber attachment, reliability testing etc. contribute to a throughput that is far less than the practical requirement [5], [6]. Thus, a significant

N. Mangal, J. Missinne, G. Van Steenberge are with the Centre for Microsystems Technology, Ghent University and imec, Zwijnaarde Technologiepark 126, 9052 Ghent, Belgium (e-mail: nivesh.mangal@imec.be, jeroen.missinne@UGent.be; geert.vanSteenberge@UGent.be)

N. Mangal, B. Snyder, J. Van Campenhout are with imec, Kapeldreef75, Heverlee B-3001, Belgium (e-mail: nivesh.mangal@imec.be, bradely.w.snyder@gmail.com; joris.vancampenhout@imec.be)

Manuscript received -; revised -. price point advantage gained from a CMOS-like wafer-scale platform, is totally lost. For example, a typical fiber attachment process on a photonics die consists of actively aligning an optical fiber array to edge couplers or grating couplers, and then glue-cure it to the die, which is time-consuming and affects the throughput of a manufacturing facility looking to ship thousands of components a day. This is because an optical signal when coupled out of a grating coupler, that is usually mode-matched to a standard single-mode fiber, at best, provides a $\pm 2.5 \mu \mathrm{m}$ lateral alignment tolerance [7] that necessitates an active alignment driven fiber-array packaging. Similarly, a photonics chip assembled via a solder-reflow based approach can easily encounter several microns of lateral displacement that can impact the coupling performance of the component if the receiving end of the coupling component is fixed (e.g. a readout system for sensing or a detector for chipto-board coupling), although some efforts have been made towards solder reflow compatibility recently [4].

Hence, a passive alignment based strategy is needed that can be low-cost, scalable, generic to any material platform, mass deployable and if possible, enable solutions for pluggable optics as well. In order to realize this, the optical mode from the coupling interface needs to be expanded to achieve a relaxed lateral alignment tolerance. As shown in Fig. 1, one can obtain a $3 \mathrm{x}$ improvement in the lateral alignment tolerance with an expanded mode diameter of $34 \mu \mathrm{m}$ when compared to a standard fiber mode diameter of $9.2 \mu \mathrm{m}$. It is also well understood that this comes at the expense of an increase in sensitivity to angular misalignment, which is however relatively easier to control than lateral misalignment with an appropriate mechanical design during assembly as long as it is above a $0.1^{\circ}$-limit [8]. For fiber-coupled lasers, beam expansion/collimation is usually achieved by integrating hemispherical lenses at the tip of the fiber [9], [10] or with the use of a ball lens [11], [12]. On-chip beam expansion has been recently demonstrated using both edge couplers with 3D-printed optics [13] and grating couplers combined with mounted microlens array blocks [14], ball-lens [15] or polymer microlens [16]. In order to truly leverage the expanded beam concept, a more scalable approach is required in which the optics can be incorporated monolithically at a wafer-scale using passive alignment.

Therefore, we have proposed to integrate microlenses at the backside of an integrated photonics chip to collimate the expanded beam resulting in a relaxed tolerance to both lateral and longitudinal misalignment (Fig. 2a) [16], [17]. Additionally, by using such a through-substrate coupling scheme, the device side (top-side) of the chip remains accessible for any additional 


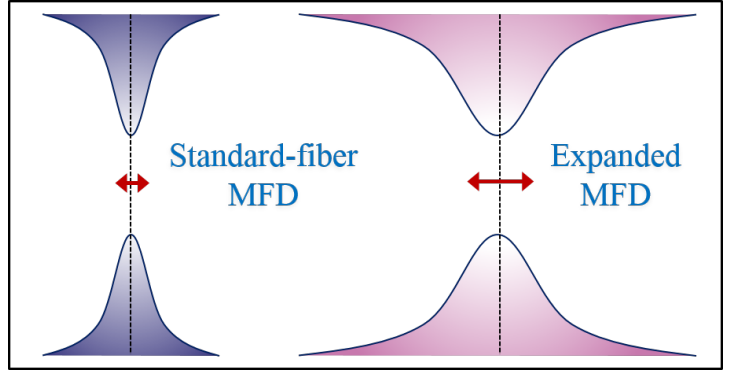

(a)
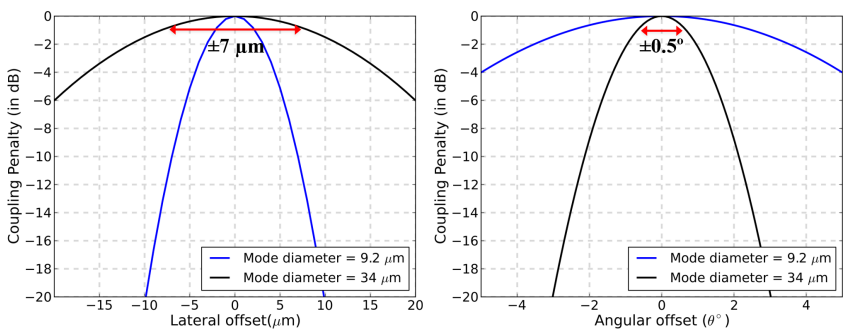

(b)

Fig. 1: An expanded mode-field diameter provides a few orders (2-3x) of improvement in lateral alignment tolerance at the expense of a reduced (0.25-0.5x) angular alignment tolerance.

fiber-array packaging and die stacking for $2.5 \mathrm{D} / 3 \mathrm{D}$ electrooptic integration (Fig. 2b). Alternatively, such an interface can be utilized for coupling to a lensed fiber array with relaxed alignment tolerances (Fig. 2c). Finally, this approach would be beneficial for various sensing applications since the device topside can be kept clear to allow the photonic circuit to interact with biomarkers or trace gas molecules. Although we have presented initial results for monolithically integrated microlenses in combination with C-band grating couplers earlier [17], the current paper (i) targets O-band grating couplers and (ii) aims at investigating in detail the tolerances and individual contributions to the coupling loss of such a backside expanded beam interface. This is achieved by hybrid integrating a silicon microlens and a photonics chip consisting of a downward directionality based O-band grating coupler. (Fig. 3).

\section{DESIGN For EXPANDED BEAM COUPLING}

The hybrid integrated photonics chip and microlens assembly is comprised of a $100 \mu \mathrm{m}$ thinned and backside polished silicon photonics chip and a separately fabricated silicon microlens array on a dual-side polished silicon substrate of 530 $\mu \mathrm{m}$ thickness (Fig. 3). The grating couplers fabricated on the topside of the chip diffract the TE-polarized waveguide mode at an angle of $2.81^{\circ}$ in silicon in a downward direction through the SOI wafer substrate. To achieve downward directionality, Ti/Al metal reflectors were deposited on the oxide cladding above the grating at a particular oxide thickness to obtain a constructive interference between the downward diffracted and metal-reflected mode. An optimal oxide thickness of $2 \mu \mathrm{m}$ between the grating and the metal reflector was used to achieve

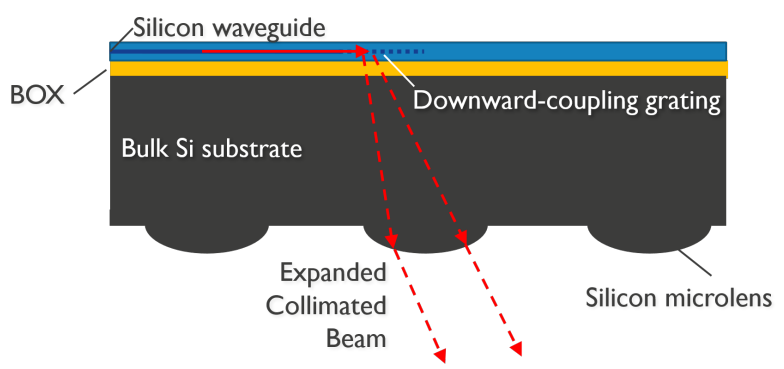

(a)

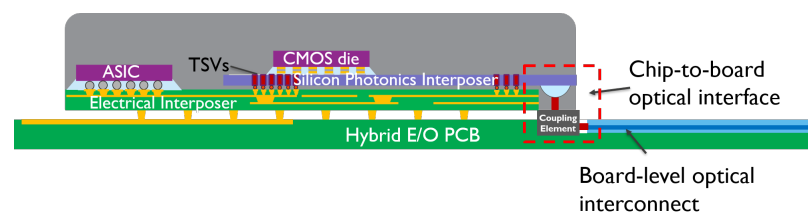

(b)

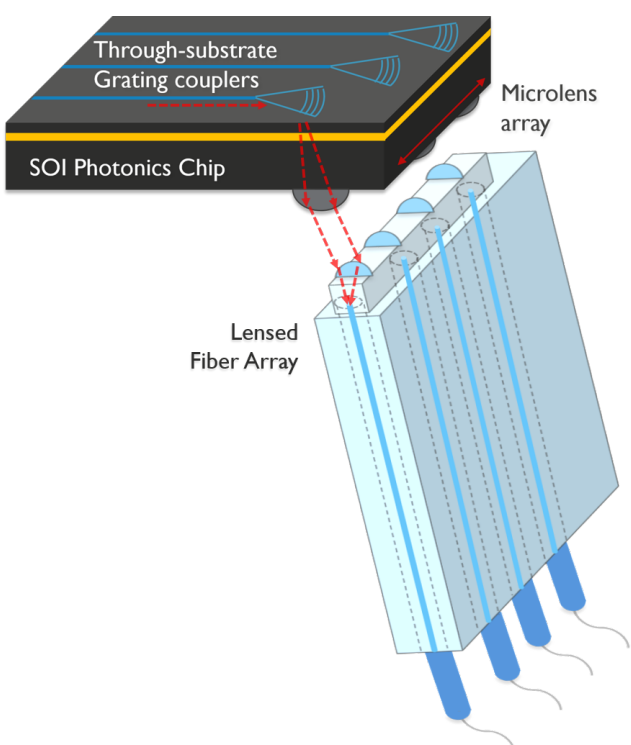

(c)

Fig. 2: (a) An overview of photonics chip expanded-beam coupling interface; (b) Application example 1: 2.5D/3D face-up integration of silicon photonics with lensed-backside coupling interface for chip-to-board coupling; (c) Application example 2: Photonics chip-to-microlensed fiber-array coupling.

this constructive interference for a wavelength of $1310 \mathrm{~nm}$ which was derived by performing $2 \mathrm{D}$ finite-difference timedomain (FDTD) simulations [18].

In order to derive the parameters of the microlens for expanded beam collimation, a ray-trace model was developed using Zemax. A $650 \mu \mathrm{m}$ thickness of the Si substrate was taken as a total thickness of the chip substrate and microlensfabricated Si substrate in the model, which allows for sufficient expansion of the incoming diffracted beam from the output grating coupler. The far-field divergence and primary angle of diffraction of the output grating coupler obtained via FDTD 


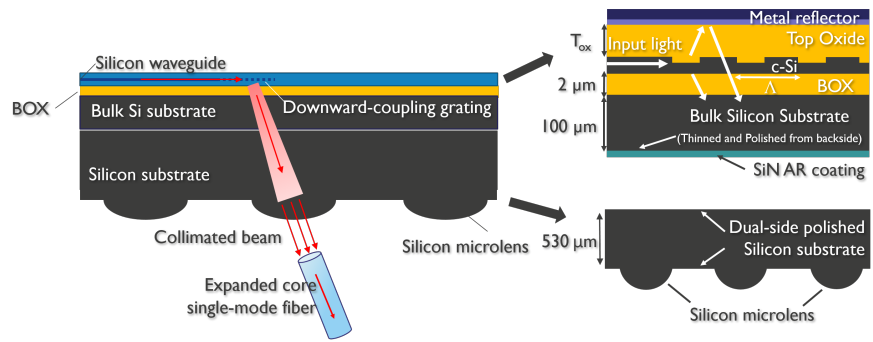

Fig. 3: Hybrid integration of silicon microlenses with a photonics chip comprised of through-substrate grating couplers. The expanded-collimated beam is coupled into a thermally expanded core single-mode fiber to investigate various alignment tolerances.

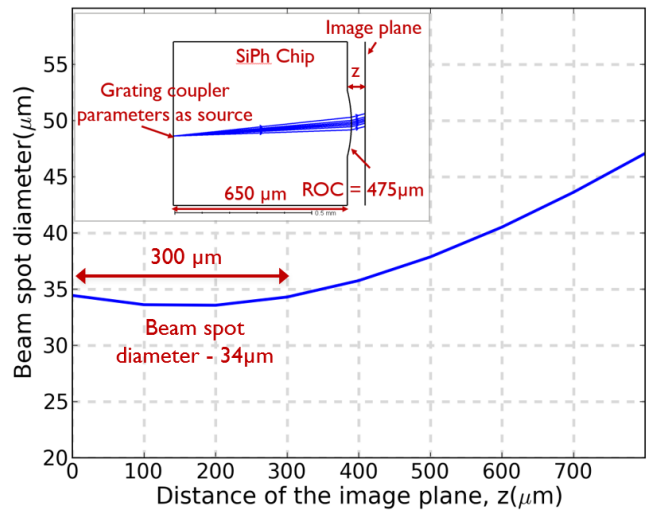

Fig. 4: A $475 \mu \mathrm{m}$ radius of curvature was determined from the ray-trace model to result in a collimated range of $300 \mu \mathrm{m}$ for a constant expanded beam diameter of $34 \mu \mathrm{m}$ (Rayleigh range $700 \mu \mathrm{m})$. From a single-mode coupling standpoint, a $300 \mu \mathrm{m}$ range provides for a relaxed longitudinal alignment tolerance for chip-to-board coupling with a negligible coupling loss.

simulations, was defined in the ray source of the model. Although the field profile from a uniformly periodic diffraction grating is exponentially decaying, it was assumed to be Gaussian in the ray-trace model. Using the beam-propagation analysis within the ray-trace model, a parameter sweep for the radius of curvature (ROC) of the lens was performed to yield an optimal value of $475 \mu \mathrm{m}$ for a lens diameter of $250 \mu \mathrm{m}$ and achieving a collimation distance of $300 \mu \mathrm{m}$ with a beam waist diameter of $34 \mu \mathrm{m}$. The Rayleigh range of the beam is about $700 \mu \mathrm{m}$, with the waist of the beam placed $150 \mu \mathrm{m}$ away from the vertex of the lens. With the typical chip-toboard distance ranging between few hundreds of microns, this scheme for expanded beam collimation can help in achieving negligible coupling loss due to reduced mode mismatch in the longitudinal (mode-propagation) direction (Fig. 4).

\section{DEVICE FABRICATION}

The photonic die used to perform the grating coupler measurement was obtained from a wafer fabricated in imec's $200 \mathrm{~mm} \mathrm{Si}$ Photonics pilot line. The die was further postprocessed to deposit $5 \mathrm{~nm} \mathrm{Ti}+200 \mathrm{~nm} \mathrm{Al}$ reflector on the
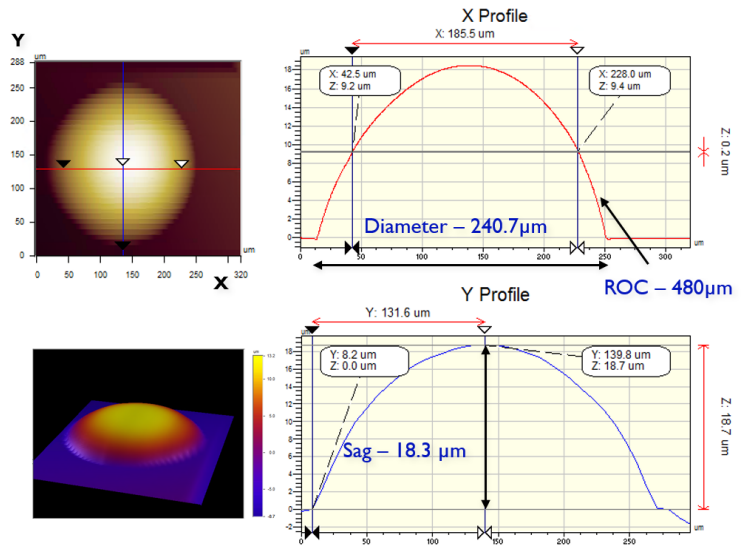

Fig. 5: A 2D surface profilometry scan of microlens post-etch was performed to determine the resulting diameter, sag and radius of curvature of the microlens.

top oxide above the grating. The die was then temporarily bonded onto a glass carrier to perform substrate-side lapping and polishing starting with a thickness of $650 \mu \mathrm{m}$ to a final value of $100 \mu \mathrm{m}$. After releasing the die from the glass carrier, a $170 \mathrm{~nm} \mathrm{SiN} \mathrm{anti-reflective(AR)} \mathrm{coating} \mathrm{was} \mathrm{deposited} \mathrm{on}$ the polished backside of the chip. For the fabrication of microlenses, Microchem AZ4562 resist was spun on top of a $530 \mu \mathrm{m}$ thick dual-side polished silicon substrate. After UVpatterning and development of the exposed resist, the obtained cylindrical structures were thermally reflowed, followed by a hard-bake. The reflown microlens profiles in resist were then transferred to silicon by a reactive-ion etch (RIE) process with an optimized composition of SF6 and O2 [17]. A 3D-surface white light profilometry was performed to verify the fabricated microlenses, which had a diameter of $240.7 \mu \mathrm{m}$, lens height (sag) of $18.3 \mu \mathrm{m}$ and a radius of curvature of $480 \mu \mathrm{m}$, which corresponded very closely to the desired specs obtained from the beam propagation simulations in the ray-trace model (Fig. 5). Also, the rms surface roughness of $\sim 10 \mathrm{~nm}$ was obtained over the fabricated microlenses in silicon.

\section{EXPERIMENT AND RESULTS}

An angled-polished fiber was glue-cured to the input grating coupler from the topside on the photonics die by performing active alignment and UV-curing NOA-61 underneath it [19]. The input fiber attachment to the photonics chip enabled us to investigate the alignment tolerance of the output grating with the fabricated microlenses. A broadband O-band SLD source was used to launch the optical signal into the input fiber. The fiber-attached photonics die was then flipped and brought underneath the substrate on which the microlenses were fabricated (Fig. 6a). A thermally expanded core singlemode fiber (TEC40 - $34 \mu \mathrm{m}$ mode field diameter) was brought close to the microlens to couple the output signal to a fiber beam-splitter connected to a power meter and an optical spectrum analyzer. A thermally expanded core (TEC) fiber has an enlarged mode field diameter obtained by locally heating a single-mode fiber at high temperature, which causes a redistribution of dopants while maintaining the condition for 


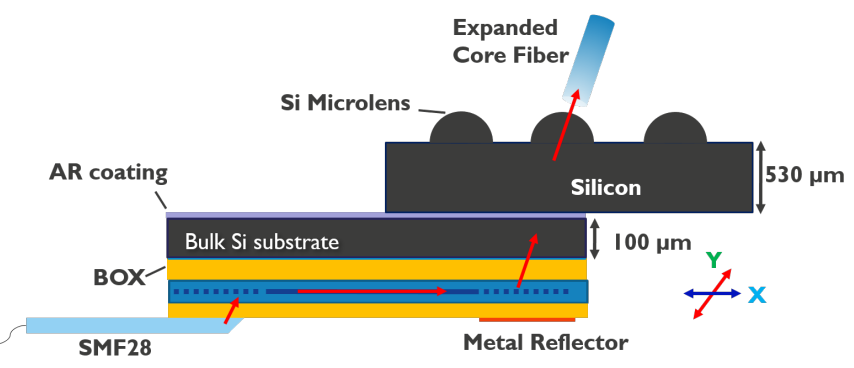

(a)
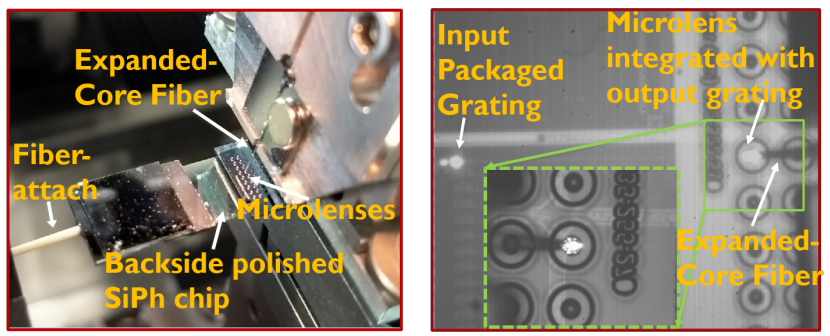

(b)

Fig. 6: (a) By actively aligning the photonics die with the far-side of the microlens-fabricated silicon substrate, coupling analysis of the optical output from the microlens with an expanded core single-mode fiber was performed; (b) (Left) An expanded-beam collimation experiment with a microlensintegrated Silicon Photonics module. (Right) IR image seen from the backside of the integrated assembly.

single-mode operation [20]. By actively aligning both the fiber and the photonic chip with respect to the microlens, an optimal coupling efficiency was obtained (Fig. 6). As shown in Fig. 7, the fiber-to-microlens coupling efficiency was compared to the case when a standard single-mode fiber (SMF28) was directly coupled to the output grating coupler from the backside of a $100 \mu \mathrm{m}$ thinned photonics chip, keeping the same input conditions on the packaged grating coupler. A $3 \mathrm{~dB}$ drop in the coupling efficiency was measured, out of which a 1.85 $\mathrm{dB}$ loss contribution was due to the Fresnel reflections from the silicon-air and fiber-air interface. This can be corrected by the use of an anti-reflective coating on the microlenses, similar to what was done on the polished backside of the photonics die. In addition, the thickness of the AR coating deposited on the backside of the photonics chip was off by -30 $\mathrm{nm}$ from the target value $(170 \mathrm{~nm})$ resulting in some ripples in the spectrum. Also, the two actively aligned components were not bonded in order to study the respective alignment tolerances. This would have resulted in the presence of a very narrow air gap between the two components contributing to some additional loss. Lastly, a decrement in the fiber-tofiber spectral bandwidth can be attributed to a low NA (0.02) of the TEC40 fiber. Although the focus of the paper is to investigate the alignment tolerances, it is interesting to tabulate the measured and estimated optical loss distribution across the expanded beam interface, listing the corrective measures needed for further improvement (Table I).

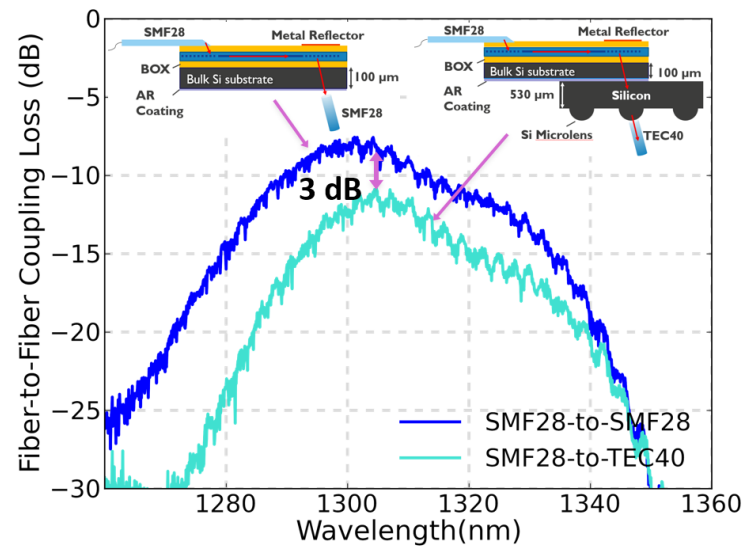

Fig. 7: A $3 \mathrm{~dB}$ drop in coupling efficiency was measured after hybrid integrating the microlens on the backside of the chip. Out of this, a $1.85 \mathrm{~dB}$ Fresnel loss occurred from the $\mathrm{Si}$ microlens-air and air-fiber interface.

TABLE I: Optical loss distribution across the expanded beam interface

\begin{tabular}{lccc}
\hline Interface & $\begin{array}{c}\text { Loss } \\
\text { (dB) }\end{array}$ & $\begin{array}{c}\text { Estimated Loss } \\
\text { After Further } \\
\text { Optimization } \\
\text { (dB) }\end{array}$ & $\begin{array}{c}\text { Corrective } \\
\text { Measures }\end{array}$ \\
\hline \hline $\begin{array}{l}\text { Fiber-attached } \\
\text { input grating }\end{array}$ & 4.5 & 2.5 & $\begin{array}{c}\text { Raised Grating } \\
\text { Couplers [21] }\end{array}$ \\
$\begin{array}{l}\text { Output Grating } \\
\text { Fresnel Loss }\end{array}$ & 3.5 & 1.5 & $\begin{array}{c}\text { Improvement in } \\
\text { directionality [22] }\end{array}$ \\
$\begin{array}{l}\text { Loss due to } \\
\text { phase mis- } \\
\text { match/microlens }\end{array}$ & 1.85 & 0 & $\begin{array}{c}\text { AR coating on } \\
\text { microlenses }\end{array}$ \\
\hline Net Loss & $\mathbf{1 1}$ & 1 & $\begin{array}{c}\text { Needs further } \\
\text { investigation }\end{array}$ \\
\hline
\end{tabular}

In order to investigate the tolerances arising out of the placement of the microlens with respect to the output grating coupler, if it were to be monolithically fabricated on the chip backside [17], a lateral alignment of the photonic chip was performed with respect to the microlens substrate, keeping the output fiber position fixed. A $\pm 2.5 \mu \mathrm{m}$ lateral 1-dB alignment tolerance was measured between the grating and the microlens (Fig. 8a). From a standpoint of fabricating microlenses monolithically on the backside of a silicon photonics wafer, a relative misalignment between the grating and microlens will result in a lateral and angular shift of the optical beam relative to its optimum coupling position, thereby contributing to an additional coupling loss and an undesirable shift in the grating coupler spectrum. In this context, a large value of $\pm 2.5 \mu \mathrm{m}$ holds good considering a sub-100 nm level overlay accuracy acceptable with the dual-sided aligners used in conventional lithography processes in wafer-scale manufacturing [23]. Next, with an optimum microlens to grating alignment, the tolerances (lateral and angular) arising out of coupling an expanded mode diameter of $34 \mu \mathrm{m}$ to a thermally expanded core fiber (TEC40) were investigated. $\mathrm{A} \pm 7 \mu \mathrm{m}$ lateral and a $\pm 0.5^{\circ}$ angular 1-dB alignment tolerance was measured between the 


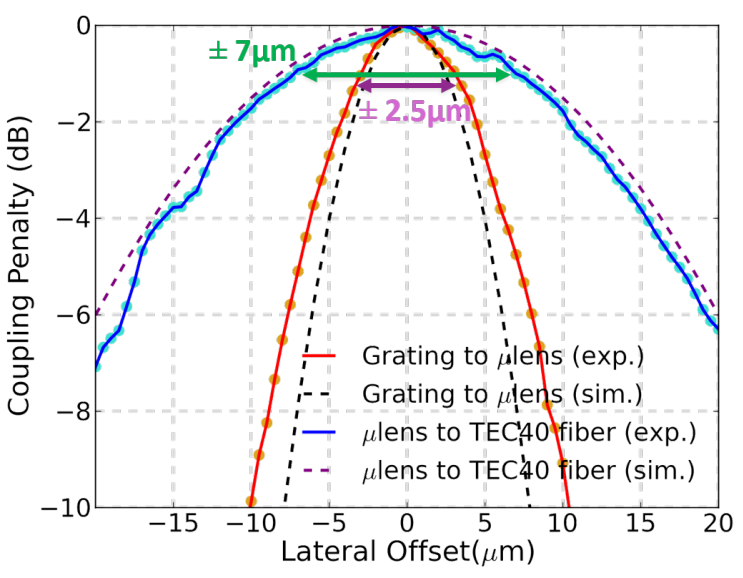

(a)

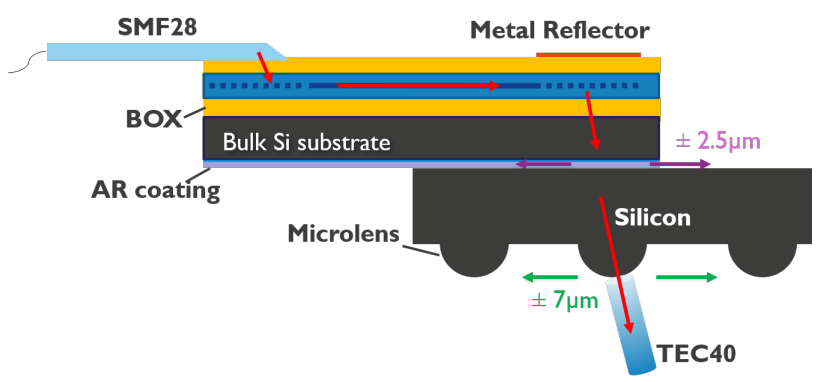

(c)

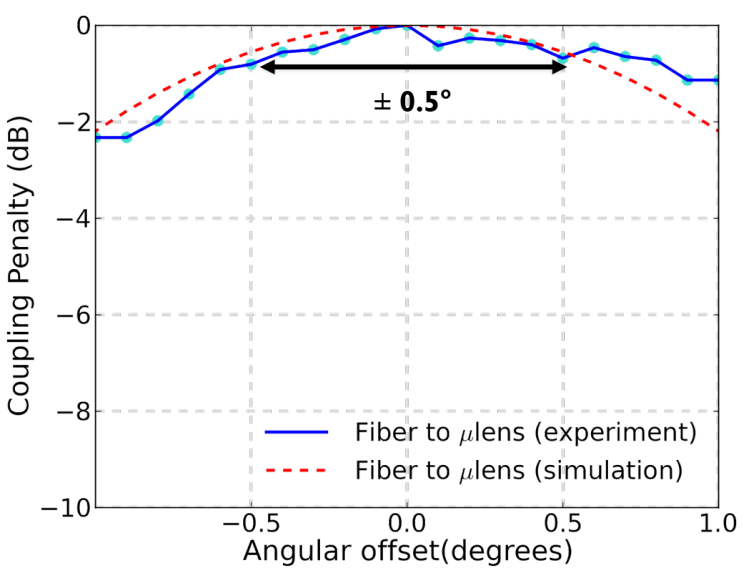

(b)

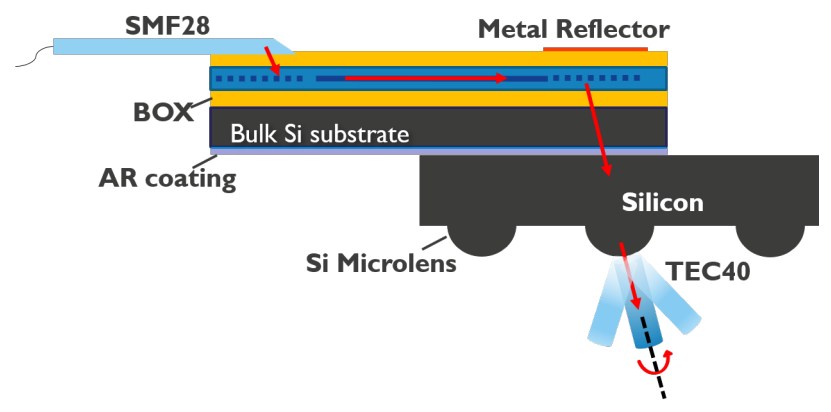

(d)

Fig. 8: (a) $\mathrm{A} \pm 7 \mu \mathrm{m}$ lateral and (b) $\pm 0.5^{\circ}$ angular 1-dB alignment tolerance was measured between an expanded mode coupled from the microlens to a single-mode expanded-core fiber; Schematics (c) and (d) illustrate the methodology of the corresponding measurements.

microlens and the expanded core fiber (Fig. 8a and 8b). A 3 -dB lateral alignment tolerance extends this limit to \pm 12.5 $\mu \mathrm{m}$. These values match well with the expected alignment tolerance (lateral and angular) simulated under the Gaussian approximation.

Lastly, the expanded core fiber was retracted away from the microlens vertex successively in steps of $100 \mu \mathrm{m}$ along the axis of the output beam and the fiber-to-microlens coupling efficiency was recorded at a fixed wavelength of $1310 \mathrm{~nm}$. Fig. 9 shows that a negligible drop in the coupling efficiency was measured over a distance of $300 \mu \mathrm{m}$, which indicates that the expanded beam was collimated. An additional point to note here is that the NA of the expanded-core fiber (TEC40) was 0.02 compared to 0.14 of a standard single-mode fiber (SMF28). This implies that an expanded core fiber would have been far more sensitive to couple to a divergent beam and hence, a drop in coupling efficiency would have been measured in the range of the specified distance here, if the optical beam from the microlens wouldn't have been collimated. Moreover, a 1-dB longitudinal alignment tolerance over a distance of $700 \mu \mathrm{m}$ was measured as expected from the Gaussian beam propagation simulations in the ray-trace model.
Hence, a longitudinal tolerance range of this magnitude makes the demonstrated coupling interface an attractive proposition to perform chip-to-board coupling for face-up integration of silicon photonics interposer in the future (Fig. 2b).

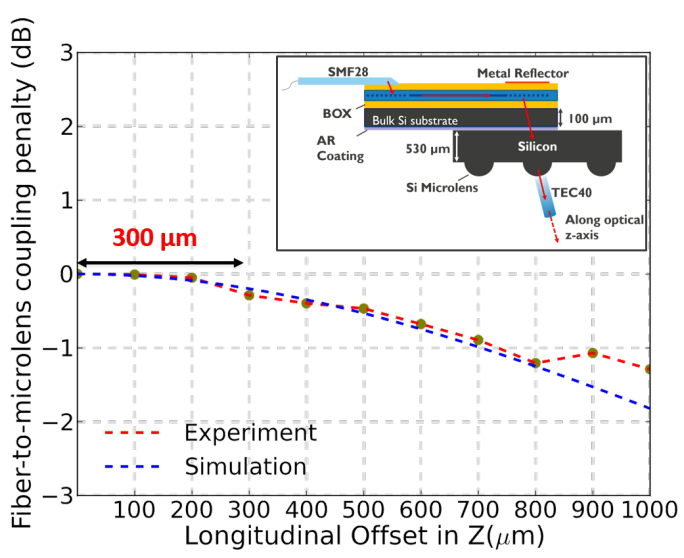

Fig. 9: By retracting the fiber along the axis of propagation of the collimated beam, a negligible drop in the coupling efficiency was measured over a distance of $300 \mu \mathrm{m}$. 


\section{CONCLUSION}

We have reported for the first time a detailed investigation of the tolerances involved in hybrid integrating silicon microlenses on the backside of a photonics chip comprised of an O-band grating coupler with downward directionality. The microlenses with a low rms roughness were fabricated on a dual-side polished silicon substrate. An expanded beam of 34 $\mu \mathrm{m}$ mode-field diameter was achieved that was collimated over a distance of $300 \mu \mathrm{m}$ with a negligible drop in coupling loss when coupled to a thermally expanded core single-mode fiber. A 1-dB fiber-to-microlens lateral and longitudinal alignment tolerances of $\pm 7 \mu \mathrm{m}$ and $700 \mu \mathrm{m}$ respectively were obtained at a wavelength of $1310 \mathrm{~nm}$. We also show that a downward directionality based expanded beam coupling interface is compatible with wafer-scale manufacturing process owing to a relatively large measured $\pm 2.5 \mu \mathrm{m} 1$-dB backside alignment accuracy between a grating and microlens. These relaxed alignment tolerance values pave the way for monolithically integrating these microlenses at the backside of a photonics chip in the near future.

\section{ACKNOWLEDGMENT}

The primary author would like to thank the entire $\mathrm{SiPh}$ integration team at imec for providing help with the silicon photonics dies fabricated from 200-mm wafer-scale processing. Acknowledgements are also extended to Filip Vermeiren and Stijn Duynslager, CMST, Ghent University for help with reactive-ion etching of microlenses and die-lapping respectively and Muhammad Muneeb, Steven Verstuyft, intec Photonics, Ghent University for help with the metal deposition and $\mathrm{SiN}$ deposition respectively. This work has been carried out as part of imec's industry affiliation program on Optical I/O, and the AQUARIUS project. The AQUARIUS project has received funding from the European Union's Horizon 2020 research and innovation programme under grant agreement No. 731465. This project is an initiative of the Photonics Public Private Partnership.

\section{REFERENCES}

[1] A. Rahim, T. Spuesens, R. Baets, and W. Bogaerts, "Open-access silicon photonics: current status and emerging initiatives," Proceedings of the IEEE, vol. 106, no. 12, pp. 2313-2330, 2018.

[2] L. Pavesi and D. J. Lockwood, Silicon photonics III: Systems and applications, Chapter 9. Springer Science \& Business Media, 2016, vol. 122.

[3] G. B. Preve, "Problems, challenges, and a critical survey on automated silicon photonics packaging and optical interconnection," in Optical Interconnects XIX, vol. 10924. International Society for Optics and Photonics, 2019, p. 1092415.

[4] T. Barwicz, T. W. Lichoulas, Y. Taira, Y. Martin, S. Takenobu, A. JantaPolczynski, H. Numata, E. L. Kimbrell, J.-W. Nah, B. Peng et al., "Breaking the mold of photonic packaging," in Integrated Optics: Devices, Materials, and Technologies XXII, vol. 10535. International Society for Optics and Photonics, 2018, p. 105350R.

[5] T. Barwicz, Y. Taira, T. W. Lichoulas, N. Boyer, Y. Martin, H. Numata, J.-W. Nah, S. Takenobu, A. Janta-Polczynski, E. L. Kimbrell et al., "A novel approach to photonic packaging leveraging existing highthroughput microelectronic facilities," IEEE Journal of Selected Topics in Quantum Electronics, vol. 22, no. 6, pp. 455-466, 2016.
[6] B. Snyder, N. Mangal, G. Lepage, S. Balakrishnan, X. Sun, N. Pantano, M. Rakowski, L. Bogaerts, P. De Heyn, P. Verheyen, A. Miller, M. Pantouvaki, P. Absil, and J. Van Campenhout, "Packaging and assembly challenges for 50G silicon photonics interposers," in 2018 Optical Fiber Communications Conference and Exposition (OFC). IEEE, 2018, pp. $1-3$.

[7] B. W. Snyder, "Hybrid integration and packaging of grating-coupled silicon photonics," Ph.D. dissertation, University College Cork, 2013.

[8] S. D. Le, P. Rochard, J.-B. Briand, L. Quétel, S. Claudot, and M. Thual, "Coupling efficiency and reflectance analysis of graded index expanded beam connectors," Journal of Lightwave Technology, vol. 34, no. 9, pp. 2092-2099, 2016.

[9] J. Kim, M. Han, S. Chang, J. W. Lee, and K. Oh, "Achievement of large spot size and long collimation length using UV curable selfassembled polymer lens on a beam expanding core-less silica fiber," IEEE Photonics Technology Letters, vol. 16, no. 11, pp. 2499-2501, 2004.

[10] J. Kim, D. Kim, B. Lee, and K. Oh, "Arrayed multimode fiber to VCSEL coupling for short reach communications using hybrid polymer-fiber lens," IEEE Photonics Technology Letters, vol. 19, no. 13, pp. 951-953, 2007.

[11] M. Sumida and K. Takemoto, "Lens coupling of laser diodes to singlemode fibers," Journal of lightwave technology, vol. 2, no. 3, pp. 305311,1984

[12] Y.-G. Lee, C.-H. Park, S.-W. Back, H.-J. Kim, and S.-S. Lee, "Alignment tolerant expanded beam connector based on a gapless fiber-lens interface," Applied optics, vol. 55, no. 2, pp. 341-344, 2016.

[13] P.-I. Dietrich, M. Blaicher, I. Reuter, M. Billah, T. Hoose, A. Hofmann, C. Caer, R. Dangel, B. Offrein, U. Troppenz et al., "In situ 3D nanoprinting of free-form coupling elements for hybrid photonic integration," Nature Photonics, vol. 12, no. 4, p. 241, 2018.

[14] C. Scarcella, K. Gradkowski, L. Carroll, J.-S. Lee, M. Duperron, D. Fowler, and P. OBrien, "Pluggable single-mode fiber-array-to-PIC coupling using micro-lenses," IEEE Photonics Technology Letters, vol. 29, no. 22, pp. 1943-1946, 2017.

[15] N. Mangal, J. Missinne, J. Van Campenhout, G. Van Steenberge, and B. Snyder, "Integration of ball lens in through-package via to enable photonic chip-to-board coupling," in 2018 IEEE 68th Electronic Components and Technology Conference (ECTC). IEEE, 2018, pp. 1140-1145.

[16] N. Mangal, J. Missinne, G. Roelkens, J. V. Campenhout, G. V. Steenberge, and B. Snyder, "Expanded-beam through-substrate coupling interface for alignment tolerant packaging of silicon photonics," in 2018 Optical Fiber Communications Conference and Exposition (OFC). IEEE, 2018, pp. 1-3.

[17] J. Missinne, N. T. Benéitez, N. Mangal, J. Zhang, A. Vasiliev, J. Van Campenhout, B. Snyder, G. Roelkens, and G. Van Steenberge, "Alignment-tolerant interfacing of a photonic integrated circuit using back side etched silicon microlenses," in Silicon Photonics XIV, vol. 10923. International Society for Optics and Photonics, 2019, p. 1092304.

[18] N. Mangal, J. Missinne, J. Van Campenhout, G. Van Steenberge, and B. Snyder, "Through-substrate coupling elements for silicon-photonicsbased short-reach optical interconnects," in Optical Interconnects XIX, vol. 10924. International Society for Optics and Photonics, 2019, p. 109240D

[19] B. Snyder and P. O'Brien, "Packaging process for grating-coupled silicon photonic waveguides using angle-polished fibers," IEEE Transactions on Components, Packaging and Manufacturing Technology, vol. 3, no. 6, pp. 954-959, 2013

[20] M. Kihara, M. Matsumoto, T. Haibara, and S. Tomita, "Characteristics of thermally expanded core fiber," Journal of lightwave technology, vol. 14, no. 10, pp. 2209-2214, 1996.

[21] G. Roelkens, D. Van Thourhout, and R. Baets, "High efficiency siliconon-insulator grating coupler based on a poly-silicon overlay," Optics Express, vol. 14, no. 24, pp. $11622-11630,2006$.

[22] N. Mangal, J. Missinne, G. Van Steenberge, J. Van Campenhout, and B. Snyder, "Performance evaluation of backside emitting O-Band grating couplers for $100 \mu \mathrm{m}$-thick silicon photonics interposers," IEEE Photonics Journal, 2019.

[23] L. Marinier, W. van Noort, R. Pellens, B. Sutedja, R. Dekker, and H. van Zeijl, "Front-to back-side overlay optimization after wafer bonding for 3D integration," Microelectronic engineering, vol. 83, no. 4-9, pp. 1229 1232,2006 
Nivesh Mangal received the MSc (Engg.) degree in the area of integrated optics from Indian Institute of Science (IISc), Bangalore in 2013. He was a Research Engr. with the computational lithography group at IBM SRDC (now GlobalFoundries Inc.), Bangalore from 2014-15. He is currently working towards the Ph.D. degree in Optical engineering with Optical I/O group, imec and Printed Photonics Group, CMST (Center for MicroSystems Technology), an imec-affiliated research lab at Ghent University, Belgium since 2015. His current research interests range from system-level optical design to passive silicon photonic devices, particularly gratings and edge couplers, fiber-chip packaging and micro-optical components for board-level optical interconnects. $\mathrm{He}$ is also a student member of IEEE Photonics Society, SPIE and OSA.

Bradley Snyder received the B.S. degree in computer engineering and the M.S. degree in electrical engineering with focus in electro-optics, both from the University of Dayton, Dayton, OH, USA, in 2002 and 2005, respectively, and the Ph.D. degree in applied physics from the Tyndall National Institute, University College Cork, Cork, Ireland, in July 2013. He was a Sr. Systems Engineer with Reed Elsevier Technology Services from 2000 to 2006, and a Software Developer with ColeSoft Marketing, Inc. from 2006 to 2009. After completing his doctoral studies, he worked as a Sr. R\&D Engineer with imec in Leuven, Belgium from 2013-2018 and currently works in a Silicon Valley photonic quantum computing startup. His principal research interests include photonic integration and packaging.

Joris Van Campenhout received the Masters degree in physics engineering, in 2002, and the Ph.D. degree in electrical engineering, in 2007, both from Ghent University, Ghent, Belgium. After his Ph.D., he worked as a Postdoctoral Researcher with the IBM T. J. Watson Research Center, New York, NY, USA. In 2010, he joined imec, Belgium, where he is currently the Program Manager, Optical I/O.

Geert Van Steenberge is a part-time professor at Ghent University, and R\&D team leader at imec. He obtained a $\mathrm{PhD}$ degree in Electrical Engineering from Ghent University in 2006, for his work on board-level parallel optical interconnects. His research interest includes on-board optics, electronicsphotonics integration, polymer and glass waveguide based optical interposers, free-space optical coupling to photonic integrated circuits, low-temperature soldering, and high-speed assembly of micro-components based on laserinduced forward-transfer. He holds 9 patents or patent applications and has authored or co-authored over 100 papers in the field of photonics packaging and integration.

Jeroen Missinne received the Master of Science degree in Electrical Engineering in 2007 and his $\mathrm{PhD}$ in Electrical Engineering in 2011, both at Ghent University, Belgium. He is currently employed as part-time professor at Ghent University and employed at the CMST (Center for MicroSystems Technology), an imec-affiliated research lab at Ghent University. His research involves photonic sensors and photonic packaging, with activities in mechanically flexible photonics, polymer optical waveguides, integration of optical and optoelectronic components, optical coupling to photonic integrated chips and laser-based technologies. 